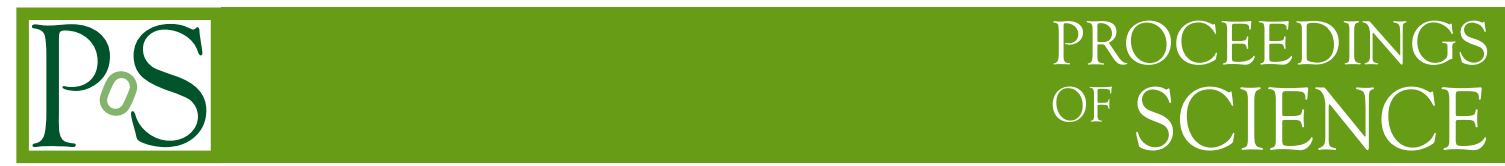

\title{
Rapidity evolution of gluon TMD from low to moderate $x$
}

\section{Andrey TARASOV}

Jefferson Lab

E-mail: atarasovejlab.org

\begin{abstract}
We study evolution of the gluon transverse momentum dependent (TMD) operator using rapidity factorization approach. The obtained rapidity evolution equation is valid at all values of the gluon longitudinal momentum fraction $x$. At small values of $x$ we apply the shock-wave approximation, while at moderate- $x$ we use the light-cone expansion formalism. The equation describes transition between linear evolution at $x \sim 1$ and non-linear one at $x \ll 1$.
\end{abstract}

XXIII International Workshop on Deep-Inelastic Scattering,

27 April - May 12015

Dallas, Texas 


\section{Introduction}

At present, methods of analysis of a non-perturbative part of hadronic scattering processes are limited. The standard way to overcome this obstacle and perform calculations in QCD is to use a factorization approach. It implies that interactions at different scales can be disentangled from each other. The non-perturbative hadron structure, in this case, is described by distribution functions, which are universal and don't depend on the process.

A good example of this is the transverse momentum dependent (TMD) distribution functions. These functions depend on longitudinal momentum fraction and the transverse momentum of the parton as well. There is a number of processes where these distributions are relevant: semiinclusive DIS, dijet production, etc. All of them involve quark TMDs. A study of this functions started three decades ago [1], but the full treatment was published only recently, see [2, 3, 4].

In recent years, the attention of community was drawn to a new class of TMD distributions with a gluon to be an active parton. That happened not only due to pure theoretical interest but the role this functions will play at future machines like EIC [5] and LHeC [6], where gluons will make the bulk of produced particles.

However, a rigorous treatment of gluon TMDs is yet to be presented. ${ }^{1}$ It should include nonlinear effects typical for small-x and contain transition to moderate-x. A subject of this notes is the gluon TMD evolution. Here you will find a summary of results from [8, 9], where a detailed analysis was presented.

\section{Definitions}

In this study we don't discuss the problem of the TMD factorization at small- $x$ and how it is connected with the moderate- $x$ approach. Instead, we start with the pure definition of the gluon TMD operator

$$
\tilde{\mathscr{F}}_{i}^{a \eta}\left(x_{\perp}, \beta_{B}\right) \mathscr{F}_{j}^{a \eta}\left(y_{\perp}, \beta_{B}\right)
$$

and consider its rapidity evolution. We are aware that the modern definition of the operator includes the so-called soft factor [3]. However, the role of this factor at small-x is not clear, so for the purpose of this work we don't include it. As a result our definition is a product of two operators with semi-infinite Wilson-lines:

$$
\mathscr{F}_{j}^{a \eta}\left(y_{\perp}, \beta_{B}\right) \equiv \frac{2}{s} \int d y_{*} e^{i \beta_{B} y_{*}}\left(\left[\infty, y_{*}\right]_{y}^{a m} g F_{\bullet j}^{m}\left(y_{*}, y_{\perp}\right)\right)^{\eta}
$$

and its complex conjugation $\tilde{\mathscr{F}}_{i}^{a \eta}\left(x_{\perp}, \beta_{B}\right)$.

For the momentum vectors we use the standard Sudakov variables: $k=\alpha p_{1}+\beta p_{2}+k_{\perp}$, where $p_{1}$ and $p_{2}$ are light-like vectors such that $p_{1}=n$ is defined by the process and $p_{2}=p-\frac{m^{2}}{s} n$, where $p$ is the momentum of the target of mass $m$. The corresponding coordinate variables are $z_{\bullet}=z_{\mu} p_{1}^{\mu}$ and $z_{*}=z_{\mu} p_{2}^{\mu}$. Connection with the usual light cone variables is very simple: $z_{\bullet}=\sqrt{\frac{s}{2}} z^{+}$and $z_{*}=\sqrt{\frac{s}{2}} z^{-}$. It is obvious that one can interpret $\beta_{B}$ as the longitudinal momentum fraction. In this study we want to obtain evolution of operator (2.1), which is correct for any value of $\beta_{B}$.

\footnotetext{
${ }^{1}$ For the study of quark TMDs in the low-x region see recent preprint [7]
} 
The Wilson-line operator

$$
\left[\infty, y_{*}\right]_{y}=\operatorname{Pexp}\left[\frac{2 i g}{s} \int_{y_{*}}^{\infty} d z_{*} p_{1}^{\mu} A_{\mu}\left(z_{*}, y_{\perp}\right)\right]
$$

corresponds to the final state interactions with the target. Note that the line goes into $+\infty$, which is relevant to the SIDIS-like process and gluon fragmentation as well. The problem with an opposite direction of the Wilson line was examined in [10].

\section{Rapidity factorization}

It is well known that there is a rapidity divergence in definition (2.1). We regularize it by a rapidity cutoff $\eta$, so the gluon fields in the definition are restricted as

$$
A_{\mu}^{\eta}(x)=\int \frac{d^{4} k}{16 \pi^{4}} \theta\left(e^{\eta}-|\alpha|\right) e^{-i k \cdot x} A_{\mu}(k) .
$$

In a sense of rapidity factorization approach [11], one should understand the cutoff as a factorization parameter between the impact factor and the matrix element. Our aim is to construct an evolution of the TMD operator with $\eta=\ln \sigma$, where $\sigma$ is a corresponding cutoff in $\alpha$.

We do it in a standard way. We shift the cutoff to a new value $\eta^{\prime}$ and integrate over "slow" fields with rapidity $\eta^{\prime}<y<\eta$, where $y=\ln \alpha$. We suppose that the "fast" fields with $y<\eta^{\prime}$ are fixed and serve as a background field. The typical diagrams are given in Fig. 1. We aim to obtain evolution at the one loop order so there is only one "quantum" (blue) line of the "slow" fields propagating in the "classical" (red) background of the "fast" fields. In calculation of this diagrams

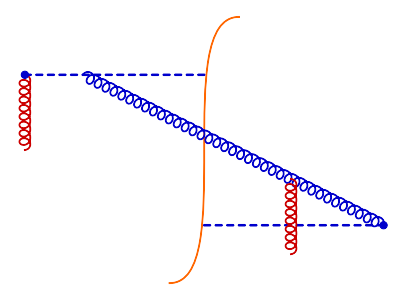

(a)

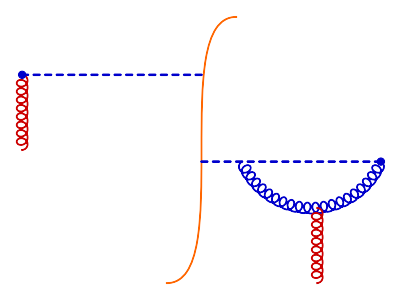

(b)

Figure 1: Typical diagrams for real (a) and virtual (b) gluon emission

we distinguish between two limits: $k_{\perp}^{2} \sim l_{\perp}^{2}$ and $k_{\perp}^{2} \gg l_{\perp}^{2}$, where $k_{\perp}$ is a transverse momentum of the "quantum" fields, while $l_{\perp}$ is the characteristic transverse scale of the external fields.

It is easy to understand that the typical longitudinal distance traveled by the "slow" fields is $\alpha s / k_{\perp}^{2} \sim \sigma s / k_{\perp}^{2}$. At the same time the longitudinal scale of the "fast" fields is $\sigma_{*} \sim \sigma^{\prime} s / l_{\perp}^{2}$. We suppose that $\sigma \gg \sigma^{\prime}$, so in the limit $k_{\perp}^{2} \sim l_{\perp}^{2}$ the "quantum" fields effectively propagate through a thin area of the "fast" fields of the background. This is the shock-wave approximation: the red (external) fields in Fig. 1 are concentrated within a segment $\left(-\sigma_{*}, \sigma\right)$. Of course this segment is very short, but in our calculation it is nevertheless finite. We consider propagation of the "quantum" (blue) fields through this segment and expand the corresponding propagator in powers of $\frac{k_{\perp}^{2}}{\alpha s} \sigma * \sim$ $\frac{\sigma^{\prime}}{\sigma} \ll 1$. 
However, in the limit $k_{\perp} \gg l_{\perp}$ this scheme is not valid: there is no separation in distance between the "slow" and "fast" fields. Insteed of the shock-wave approximation, in this case we apply the light-cone expansion approach [12]. We take a ration $l_{\perp} / k_{\perp} \ll 1$ as an expansion parameter and expand the gluon propagator in Fig. 1 around the light ray $y_{\perp}+\frac{2}{s} y_{*} p_{1}$, which is the direction of the Wilson line in definition (2.1).

\section{The Lipatov vertex}

As usual, there are two contributions into the evolution kernel: the real gluon emission (Fig. 1a) and the virtual diagrams (Fig. 1b). The "real" correction to the TMD operator (2.1) is

$$
\left\langle\tilde{\mathscr{F}}_{i}^{a}\left(x_{\perp}, \beta_{B}\right) \mathscr{F}_{j}^{a}\left(y_{\perp}, \beta_{B}\right)\right\rangle^{\ln \sigma}=-\int_{\sigma^{\prime}}^{\sigma} \frac{d \alpha}{2 \alpha} d^{2} k_{\perp}\left(\tilde{L}_{i}^{b a ; \mu}\left(k, x_{\perp}, \beta_{B}\right) L_{\mu j}^{a b}\left(k, y_{\perp}, \beta_{B}\right)\right)^{\ln \sigma^{\prime}},
$$

where the Lipatov vertex $L_{\mu i}^{a b}\left(k, y_{\perp}, \beta_{B}\right)=i \lim _{k^{2} \rightarrow 0} k^{2}\left\langle A_{\mu}^{a}(k) \mathscr{F}_{i}^{b}\left(y_{\perp}, \beta_{B}\right)\right\rangle$ is an amplitude of the single-gluon production by the operator $\mathscr{F}_{i}^{b}$. There are several possible types of emission. The corresponding diagrams can be found in Fig. 2. We start with the calculation of the Lipatov vertex

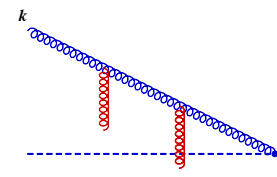

(a)



(b)

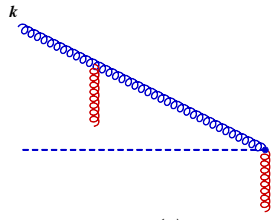

(c)

Figure 2: Diagrams contributing into the Lipatov vertex

in the light-cone approach. We expand the gluon propagator around direction of the Wilson line and neglect terms suppressed by $l_{\perp} / k_{\perp} \ll 1$. One can show that the leading contribution comes from the terms linear in the gluon strength tensor $F_{\bullet} i$. The external (red) field has no specific longitudinal properties and we take into account all possible emissions from the Wilson line and the strength tensor in the definition (2.1) as well. The details of the calculation can be found in [9]. Here we show only final result:

$$
\begin{aligned}
& L_{\mu i}^{a b}\left(k, y_{\perp}, \beta_{B}\right)=i \lim _{k^{2} \rightarrow 0} k^{2}\left\langle A_{\mu}^{a \mathrm{q}}(k)\left(\mathscr{F}_{i}^{b}\left(\beta_{B}, y_{\perp}\right)\right)^{1 \mathrm{st}}\right\rangle=\frac{2 g e^{-i(k, y)_{\perp}}}{\alpha \beta_{B} s+k_{\perp}^{2}} \\
& \times\left[\frac{\alpha \beta_{B} s}{k_{\perp}^{2}}\left(\frac{k_{\perp}^{2}}{\alpha s} p_{2 \mu}-\alpha p_{1 \mu}\right) \delta_{i}^{l}-\delta_{\mu}^{l} k_{i}+\frac{\alpha \beta_{B} g_{\mu i} k^{l}}{k_{\perp}^{2}+\alpha \beta_{B} s}+\frac{2 \alpha k_{i} k^{l}}{k_{\perp}^{2}+\alpha \beta_{B} s} p_{1 \mu}\right] \mathscr{F}_{l}^{a b}\left(\beta_{B}+\frac{k_{\perp}^{2}}{\alpha s}, y_{\perp}\right) .
\end{aligned}
$$

On the other hand, in the limit $k_{\perp} \sim l_{\perp}$ we apply the shock-wave approximation. In this case the external field in Fig. 2 shrinks to fill a small area from $-\sigma_{*}$ to $\sigma_{*}$ in longitudinal direction. Effectively the diagrams in Fig. 2 take a new look, which is presented in Fig. 3.

Now we take into account not only different types of emission, but the position of the emission point as well: we separate the emission from the left, right sides and from inside of the shock-wave. The sum of all diagrams in Fig. 3 is

$$
L_{\mu i}^{a b}\left(k, y_{\perp}, \beta_{B}\right)=2 g e^{-i(k, y) \perp}\left(\frac{p_{2 \mu}}{\alpha s}-\frac{\alpha p_{1 \mu}}{k_{\perp}^{2}}\right)\left[\mathscr{F}_{i}\left(\beta_{B}, y_{\perp}\right)-U_{i}\left(y_{\perp}\right)\right]^{a b}
$$




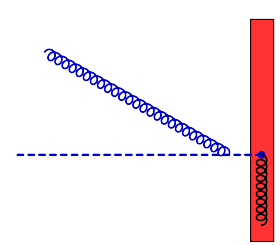

(a)

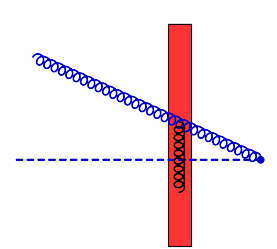

(b)

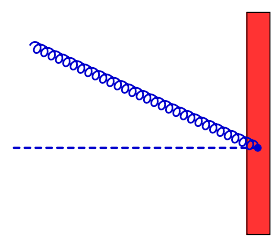

(c)

Figure 3: Lipatov vertex in the shock-wave approximation

$$
\begin{aligned}
& +g\left(k_{\perp} \mid g_{\mu i}\left(\frac{\alpha \beta_{B} s}{\alpha \beta_{B} s+p_{\perp}^{2}}-U \frac{\alpha \beta_{B} s}{\alpha \beta_{B} s+p_{\perp}^{2}} U^{\dagger}\right)+2 \alpha p_{1 \mu}\left(\frac{p_{i}}{\alpha \beta_{B} s+p_{\perp}^{2}}-U \frac{p_{i}}{\alpha \beta_{B} s+p_{\perp}^{2}} U^{\dagger}\right)\right. \\
& \left.+\left[2 i \beta_{B} p_{2 \mu} \partial_{i} U-2 i \partial_{\mu}^{\perp} U p_{i}+\frac{2 p_{2 \mu}}{\alpha s} \partial_{\perp}^{2} U p_{i}\right] \frac{1}{\alpha \beta_{B} s+p_{\perp}^{2}} U^{\dagger}-\frac{2 \alpha p_{1 \mu}}{p_{\perp}^{2}} U_{i} \mid y_{\perp}\right)^{a b} .
\end{aligned}
$$

In this calculation we expand the "slow" gluon propagator in powers of the size of the shock-wave and neglect terms suppressed by $\frac{p_{\perp}^{2}}{\alpha s} \sigma_{*} \ll 1$.

The form of the Lipatov vertex in the limit $k_{\perp} \gg l_{\perp}$, see (4.2), and $k_{\perp} \sim l_{\perp}$, see (4.3), looks very different. For example, (4.2) is linear in the gluon strength tensor, while (4.3) has non-linear contributions, like $\partial_{\perp}^{2} U$. However, we show that it is possible to combine two results and write an interpolation formula:

$$
\begin{aligned}
& L_{\mu i}^{a b}\left(k, y_{\perp}, \beta_{B}\right) \\
& =g\left(k_{\perp} \mid \frac{\alpha \beta_{B} s g_{\mu i}+2 \alpha p_{1 \mu} k_{i}}{\alpha \beta_{B} s+k_{\perp}^{2}}\left(2 i k^{j} \partial_{j} U-\partial_{\perp}^{2} U\right) \frac{1}{\alpha \beta_{B} s+p_{\perp}^{2}} U^{\dagger}+2 i \alpha p_{1 \mu} \partial_{i} U \frac{1}{\alpha \beta_{B} s+p_{\perp}^{2}} U^{\dagger}\right. \\
& \left.+\frac{2 i}{\alpha s} p_{2 \mu} \partial_{i} U \frac{\alpha \beta_{B} s}{\alpha \beta_{B} s+p_{\perp}^{2}} U^{\dagger}-\left[2 i \partial_{\mu} U-\frac{2 p_{2 \mu}}{\alpha s} \partial_{\perp}^{2} U\right] \frac{p_{i}}{\alpha \beta_{B} s+p_{\perp}^{2}} U^{\dagger}-\frac{2 \alpha p_{1 \mu}}{p_{\perp}^{2}} i\left(\partial_{i} U\right) U^{\dagger} \mid y_{\perp}\right)^{a b} \\
& +\frac{2 g e^{-i(k, y)_{\perp}}}{\alpha \beta_{B} s+k_{\perp}^{2}}\left[-\delta_{\mu}^{j} k_{i}+\frac{2 \alpha k_{i} k^{j} p_{1 \mu}}{\alpha \beta_{B} s+k_{\perp}^{2}}+\frac{\alpha \beta_{B} s g_{\mu i} k^{j}}{\alpha \beta_{B} s+k_{\perp}^{2}}+\beta_{B} p_{2 \mu} \delta_{i}^{j}-\alpha p_{1 \mu} \frac{\alpha \beta_{B} s}{k_{\perp}^{2}} \delta_{i}^{j}\right] \\
& \times\left[\mathscr{F}_{j}\left(\beta_{B}+\frac{k_{\perp}^{2}}{\alpha s}, y_{\perp}\right)-U_{j}\left(y_{\perp}\right)\right]^{a b} .
\end{aligned}
$$

We show that in the limit $k_{\perp} \gg l_{\perp}$ this expression simplifies to (4.2), while for $k_{\perp} \sim l_{\perp}$ it reproduces formula (4.3). We prove that it is correct in the whole region of values of $\beta_{B}$. It is also important that (4.4) doesn't break the gauge invariance: $k^{\mu} L_{\mu i}^{a b}\left(k, y_{\perp}, \beta_{B}\right)=0$. Finally, we use the Lipatov vertex (4.4) to calculate the "real" part of the evolution kernel according to (4.1).

\section{The virtual emission}

The second part of the evolution kernel is the virtual correction, see Fig. 1b. For calculation of this diagrams we apply the same strategy we used in derivation of the Lipatov vertex. In the limit $k_{\perp} \gg l_{\perp}$ we expand the gluon propagator around $y_{\perp}+\frac{2}{s} y_{*} p_{1}$ direction and neglect terms suppressed by $l_{\perp} / k_{\perp} \ll 1$. What is left is used in the explicit calculation of the diagrams if Fig. $1 \mathrm{~b}$. Note that there are actually several of them, for details see [9]. 


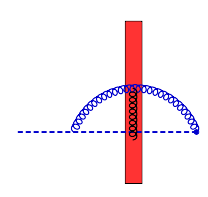

(a)



(b)

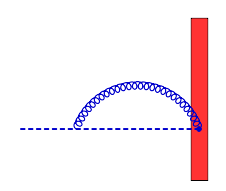

(c)

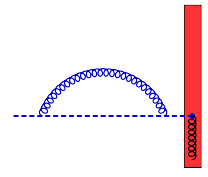

(d)

Figure 4: The virtual correction in the shock-wave approximation

In the limit $k_{\perp} \sim l_{\perp}$ we use the shock-wave approximation so the virtual emission diagrams take a form, which is given in Fig. 4. We show that the sum of this diagrams can be combined with the result obtained at $k_{\perp} \gg l_{\perp}$ and one can write a unified expression, which describes both limits and is valid for all values of $\beta_{B}$ :

$$
\begin{aligned}
& \left\langle\mathscr{F}_{i}^{n}\left(y_{\perp}, \beta_{B}\right)\right\rangle^{\mathrm{virt}}= \\
& =-i g^{2} f^{n k l} \int_{\sigma^{\prime}}^{\sigma} \frac{d \alpha}{\alpha}\left(y_{\perp}\left|\frac{p^{j}}{p_{\perp}^{2}}\left(2 \partial_{i} \partial_{j} U+g_{i j} \partial_{\perp}^{2} U\right) \frac{1}{\alpha \beta_{B} s+p_{\perp}^{2}} U^{\dagger}+\frac{\alpha \beta_{B} s p_{\perp}^{-2}}{\alpha \beta_{B} s+p_{\perp}^{2}} \mathscr{F}_{i}\left(\beta_{B}\right)\right| y_{\perp}\right)^{k l} .
\end{aligned}
$$

\section{Evolution equation for gluon TMD}

Now we can write the one-loop correction to the operator (2.1). It is a sum of the product of two Lipatov vertexes (4.4) and the virtual contribution (5.1):

$$
\begin{aligned}
& \left\langle\tilde{\mathscr{F}}_{i}^{a}\left(x_{\perp}, \beta_{B}\right) \mathscr{F}_{j}^{a}\left(y_{\perp}, \beta_{B}\right)\right\rangle_{\text {one-loop }}^{\ln \sigma}=-\int_{\sigma^{\prime}}^{\sigma} \frac{d \alpha}{2 \alpha} d^{2} k_{\perp}\left(\tilde{L}_{i}^{b a ; \mu}\left(k, x_{\perp}, \beta_{B}\right) L_{\mu j}^{a b}\left(k, y_{\perp}, \beta_{B}\right)\right)^{\ln \sigma^{\prime}} \\
& +\tilde{F}_{i}^{a}\left(x_{\perp}, \beta_{B}\right)\left\langle\mathscr{F}_{j}^{a}\left(y_{\perp}, \beta_{B}\right)\right\rangle^{\text {virt }}+\left\langle\tilde{F}_{i}^{a}\left(x_{\perp}, \beta_{B}\right)\right\rangle^{\text {virt } \mathscr{F}_{j}^{a}\left(y_{\perp}, \beta_{B}\right) .}
\end{aligned}
$$

To get the evolution equation we differentiate this expression over $\sigma$. Combining all results together we get

$$
\begin{aligned}
& \frac{d}{d \log \sigma}\left\langle p\left|\tilde{\mathscr{F}}_{i}^{a}\left(x_{\perp}, \beta_{B}\right) \mathscr{F}_{j}^{a}\left(y_{\perp}, \beta_{B}\right)\right| p\right\rangle \\
& =-\alpha_{s}\langle p| \operatorname{Tr}\left\{\int d ^ { 2 } k _ { \perp } \theta ( 1 - \beta _ { B } - \frac { k _ { \perp } ^ { 2 } } { \sigma s } ) \left[\left(x_{\perp} \mid\left(\tilde{U} \frac{1}{\sigma \beta_{B} s+p_{\perp}^{2}}\left(\tilde{U}^{\dagger} k_{k}+p_{k} \tilde{U}^{\dagger}\right)\right.\right.\right.\right. \\
& \left.\left.\times \frac{\sigma \beta_{B} s g_{\mu i}-2 k_{\mu}^{\perp} k_{i}}{\sigma \beta_{B} s+k_{\perp}^{2}}-2 k_{\mu}^{\perp} g_{i k} \tilde{U} \frac{1}{\sigma \beta_{B} s+p_{\perp}^{2}} \tilde{U}^{\dagger}-2 g_{\mu k} \tilde{U} \frac{p_{i}}{\sigma \beta_{B} s+p_{\perp}^{2}} \tilde{U}^{\dagger}\right) \tilde{F}^{k}\left(\beta_{B}+\frac{k_{\perp}^{2}}{\sigma s}\right) \mid k_{\perp}\right) \\
& \times\left(k_{\perp} \mid \mathscr{F}^{l}\left(\beta_{B}+\frac{k_{\perp}^{2}}{\sigma s}\right)\left(\frac{\sigma \beta_{B} s \delta_{j}^{\mu}-2 k_{\perp}^{\mu} k_{j}}{\sigma \beta_{B} s+k_{\perp}^{2}}\left(k_{l} U+U p_{l}\right) \frac{1}{\sigma \beta_{B} s+p_{\perp}^{2}} U^{\dagger}\right.\right. \\
& \left.\left.-2 k_{\perp}^{\mu} g_{j l} U \frac{1}{\sigma \beta_{B} s+p_{\perp}^{2}} U^{\dagger}-2 \delta_{l}^{\mu} U \frac{p_{j}}{\sigma \beta_{B} s+p_{\perp}^{2}} U^{\dagger}\right) \mid y_{\perp}\right) \\
& +2\left(x_{\perp}\left|\tilde{F}_{i}\left(\beta_{B}+\frac{k_{\perp}^{2}}{\sigma s}\right)\right| k_{\perp}\right)\left(k_{\perp} \mid \mathscr{F}^{l}\left(\beta_{B}+\frac{k_{\perp}^{2}}{\sigma s}\right)\left(\frac{k_{j}}{k_{\perp}^{2}} \frac{\sigma \beta_{B} s+2 k_{\perp}^{2}}{\sigma \beta_{B} s+k_{\perp}^{2}}\left(k_{l} U+U p_{l}\right) \frac{1}{\sigma \beta_{B} s+p_{\perp}^{2}} U^{\dagger}\right.\right. \\
& \left.\left.+2 U \frac{g_{j l}}{\sigma \beta_{B} s+p_{\perp}^{2}} U^{\dagger}-2 \frac{k_{l}}{k_{\perp}^{2}} U \frac{p_{j}}{\sigma \beta_{B} s+p_{\perp}^{2}} U^{\dagger}\right) \mid y_{\perp}\right) \\
& +2\left(x_{\perp} \mid\left(\tilde{U} \frac{1}{\sigma \beta_{B} s+p_{\perp}^{2}}\left(\tilde{U}^{\dagger} k_{k}+p_{k} \tilde{U}^{\dagger}\right) \frac{k_{i}}{k_{\perp}^{2}} \frac{\sigma \beta_{B} s+2 k_{\perp}^{2}}{\sigma \beta_{B} s+k_{\perp}^{2}}+2 \tilde{U} \frac{g_{i k}}{\sigma \beta_{B} s+p_{\perp}^{2}} \tilde{U}^{\dagger}\right.\right.
\end{aligned}
$$




$$
\begin{aligned}
& \left.\left.\left.-2 \tilde{U} \frac{p_{i}}{\sigma \beta_{B} s+p_{\perp}^{2}} \tilde{U}^{\dagger} \frac{k_{k}}{k_{\perp}^{2}}\right) \tilde{\mathscr{F}}^{k}\left(\beta_{B}+\frac{k_{\perp}^{2}}{\sigma s}\right) \mid k_{\perp}\right)\left(k_{\perp}\left|\mathscr{F}_{j}\left(\beta_{B}+\frac{k_{\perp}^{2}}{\sigma s}\right)\right| y_{\perp}\right)\right] \\
& +2 \tilde{\mathscr{F}_{i}}\left(x_{\perp}, \beta_{B}\right)\left(y_{\perp}\left|-\frac{p^{m}}{p_{\perp}^{2}} \mathscr{F}_{k}\left(\beta_{B}\right)\left(i \overleftarrow{\partial}_{l}+U_{l}\right)\left(2 \delta_{m}^{k} \delta_{j}^{l}-g_{j m} g^{k l}\right) U \frac{1}{\sigma \beta_{B} s+p_{\perp}^{2}} U^{\dagger}\right| y_{\perp}\right) \\
& +2\left(x_{\perp}\left|\tilde{U} \frac{1}{\sigma \beta_{B} s+p_{\perp}^{2}} \tilde{U}^{\dagger}\left(2 \delta_{i}^{k} \delta_{m}^{l}-g_{i m} g^{k l}\right)\left(i \partial_{k}-\tilde{U}_{k}\right) \tilde{\mathscr{F}}_{l}\left(\beta_{B}\right) \frac{p^{m}}{p_{\perp}^{2}}\right| x_{\perp}\right) \mathscr{F}_{j}\left(y_{\perp}, \beta_{B}\right) \\
& -4 \int \frac{d^{2} k_{\perp}}{k_{\perp}^{2}}\left[\theta\left(1-\beta_{B}-\frac{k_{\perp}^{2}}{\sigma s}\right) \tilde{\mathscr{F}}_{i}\left(x_{\perp}, \beta_{B}+\frac{k_{\perp}^{2}}{\sigma s}\right) \mathscr{F}_{j}\left(y_{\perp}, \beta_{B}+\frac{k_{\perp}^{2}}{\sigma s}\right) e^{i(k, x-y)}\right)_{\perp} \\
& \left.\left.-\frac{\sigma \beta_{B} s}{\sigma \beta_{B} s+k_{\perp}^{2}} \tilde{\mathscr{F}}_{i}\left(x_{\perp}, \beta_{B}\right) \mathscr{F}_{j}\left(y_{\perp}, \beta_{B}\right)\right]\right\}|p\rangle+O\left(\alpha_{s}^{2}\right) .
\end{aligned}
$$

The formula (6.2) is the main result of our study. It is an evolution equation of the gluon TMD operator (2.1) and is valid for all (except too small) values of transverse momentum $k_{\perp} \sim$ $\left(x_{\perp}-y_{\perp}\right)^{-1}$ and longitudinal momentum fraction variable $\beta_{B}$.

This result contains different kind of dynamics typical for different values of the kinematic variables. We show, that with a proper approximations this equation reproduces a set of known limits. At $x \sim 1$ (in our notation $x$ is equivalent to $\beta_{B}$ ) and large transverse momentum it yields the linear moderate $x$ dynamics with the DGLAP equation in the collinear case. At $x \sim 1$ and small $k_{\perp}$ we get the Sudakov double logarithm evolution. While at small- $x$ it reduces to the non-linear equation of the Balitsky-Kovchegov (BK) type.

The author is grateful to G.A. Chirilli, J.C. Collins, Yu. Kovchegov, A. Prokudin, A.V. Radyushkin, T. Rogers, M.D. Sievert and F. Yuan for valuable discussions. This work was supported by contract DE-AC05-06OR23177 under which the Jefferson Science Associates, LLC operates the Thomas Jefferson National Accelerator Facility.

\section{References}

[1] J. C. Collins, D. E. Soper Nucl. Phys. B194, 445 (1982).

[2] X. Ji, Jian-Ping Ma, and F. Yuan, Phys. Rev. D71, 034005 (2005).

[3] J. C. Collins, Foundations of Perturbative QCD (Cambridge University Press, Cambridge, 2011).

[4] M.G. Echevarria, A. Idilbi, and I. Scimeni, JHEP 07, 002 (2012).

[5] A. Accardi et al., "Electron Ion Collider: The Next QCD Frontier", arXiv:1212.1701.

[6] J. L. Abelleira Fernandez et al., J. Phys. G 39, 075001 (2012).

[7] Yu.V. Kovchegov and M.D. Sievert, "Calculating TMDs of an Unpolarized Target: Quasi-Classical Approximation and Quantum Evolution", arXiv:1505.01176.

[8] I. Balitsky, and A. Tarasov, Int. J. Mod. Phys. Conf. Ser. 37, 1560058 (2015).

[9] I. Balitsky, and A. Tarasov, "Rapidity evolution of gluon TMD from low to moderate x", arXiv:1505.0215.

[10] A. H. Mueller, Bo-Wen Xiao, and F. Yuan, Phys. Rev. D88, 114010 (2013); Phys. Rev. Lett. 110, 082301 (2013).

[11] I. Balitsky, Nucl. Phys. B463, 99 (1996); Phys. Rev. D60, 014020 (1999).

[12] I. Balitsky, Phys. Lett. B124, 230 (1983); I. Balitsky and V.M. Braun, Nucl. Phys. B311, 541 (1989). 